\title{
Study on a New Method for Improving Resolution of Grating Interferometer Displacement Measuring System
}

\author{
Lun Shi ${ }^{1,}$ a, He Wang, Chi-Ping Wang and Jia-Bao Chen \\ School of Mechanical Engineering, Shanghai Jiao Tong University, Shanghai, China
}

\begin{abstract}
A method for improving resolution of grating interferometer displacement measuring system is developed in this paper. The measurement system consists of a grating interferometer and a phase-shift device. Two interference fringes are required in the detecting field of photoelectric sensor. If we change the signal phase of the interference fringe by moving the photoelectric sensor between the two interference fringes, the displacement equivalent converted from the photoelectric sensor will changed. The method is applied in positioning system and the experiments' results indicate that the new displacement measuring method will improve the positioning accuracy about $33 \%$. Also, the errors in the positioning process with the new method are discussed in briefly.
\end{abstract}

Key words. Grating interferometer; phase shift; displacement

\section{Introduction}

Displacement measuring system based on grating interferometer is often used in traditional precision positioning process ${ }^{[1,2]}$. Although laser interferometer is better than the former in measurement resolution, it is limited in application sometimes because of its strict requirements on temperature, humidity and so on ${ }^{[3-5]}$. And coarse/fine dual-stage manipulators are widely studied and applied for long distance movement and high precision, ${ }^{[6-9]}$ though following with complicated mechanisms. Therefore, grating interferometer is selected as the study object in this paper. Usually the measuring resolution of grating interferometer is no better than 0.5 micrometer and it maybe reach to 0.1 micrometer or even smaller if the grating stripe signals are treated with subdivision method, whose subdivision level is limited by the stripe quality ${ }^{[10]}$. In this paper, a phase shift method applied on grating interferometer stripe is developed and it will get higher displacement measuring resolution in ultra-precision positioning system.

\section{Principle of grating interferometer measuring}

The whole displacement measuring system with grating

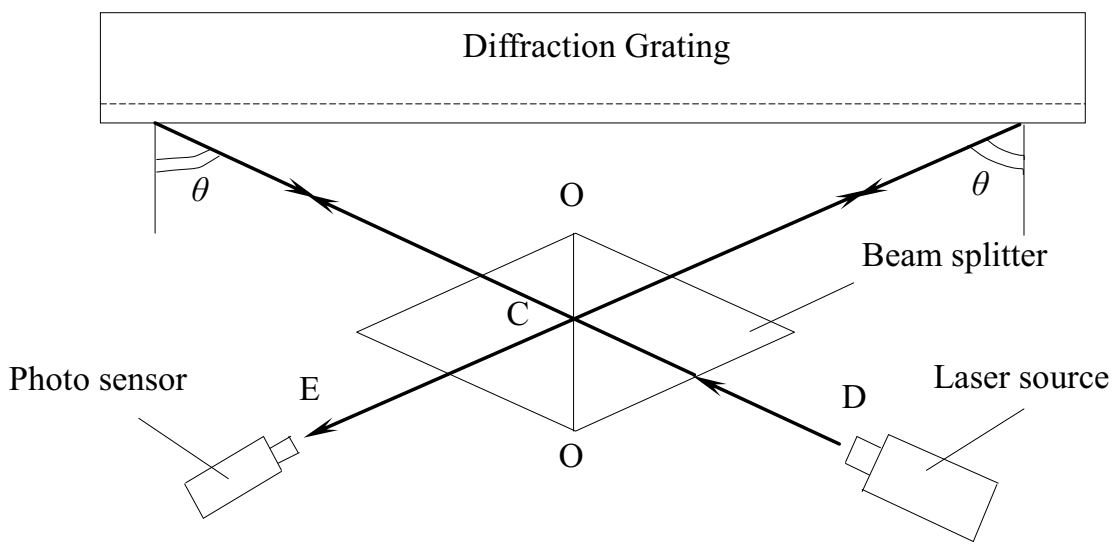

Fig. 1 Schematic of grating interferometer

Corresponding author: Lun Shi, Associate professor of Shanghai Jiao Tong University, email: shilun@sjtu.edu.cn The study is supported by NSFC ( 51275306 ) 
interferometer consists of a laser device, a blazed diffraction grating, a beam splitter with equal optical path and a photoelectric sensor, as shown in Fig.1. The parallel incident light launched from the laser device will be split into two beams, transmitted light and reflected light, when it meets the plane $\mathrm{O}-\mathrm{O}$ of the splitter. When the diffraction grating is in the case of auto-collimation, the two beams will be both diffracted back and will meet together at point $\mathrm{C}$. Therefore, the interference fringes will appear in the place where the two diffraction beams overlap.

The diffraction grating as reference grating is mounted on a linear table. When the linear table moves, the interference fringes will be light and shade in circle and it will be detected and converted into pulse signal by the sensor placed at E point. Therefore, we can control the motion of the linear table by counting the fringe pulses from the interferometer. When the reference grating (or linear table) shifts $\Delta x$ along the direction shown in Fig. 1, the phase variation of the two coherent light beams is ${ }^{[11]}$ :

$$
\Delta \Phi=4 \pi m \frac{\Delta x}{A}
$$

Where $m$ is the diffraction order and $A$ is the space of the reference grating (for example $1 / 300 \mathrm{~mm}$ ).

If $\Delta x=A$ and $m=2$, then $\Delta \Phi=2 \pi \times 4$

Namely, when the reference grating moves one space, there will be 4 fringe-signals. Therefore, the equivalent displacement to one fringe is:

$$
a^{\prime}=\frac{A}{2 m}
$$

\section{Fringe phase shift principle}

It is important to ensure that there are only two interference fringes, whose distance between them is $w$, in the detecting field illustrated in Fig 2. The $w$ value can be changed by adjusting the interferometer. It is regarded that the fringe phase signal is divided into unlimited parts from $0^{\circ}$ to $360^{\circ}$ in electrical degree and the parts are

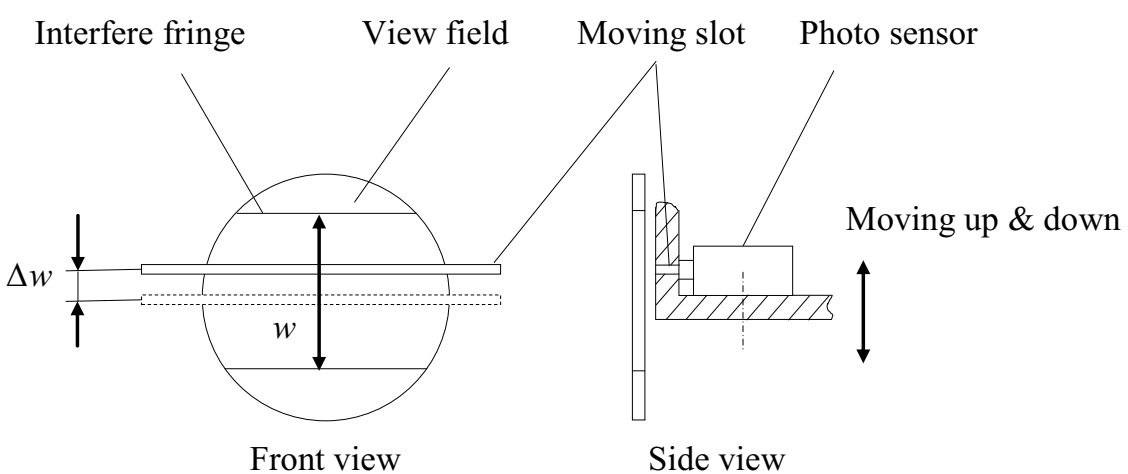

Fig. 2 Phase shift of interference fringe

distributed averagely between the two fringes in theory. Therefore, if we change the position of the photoelectric sensor, the phase of the fringe signal will be changed correspondingly.
Therefore, the increment of fringe signal can be expressed as:

$$
d a= \pm \frac{\Delta w}{w} \cdot a^{\prime}
$$

Where, $\Delta w$----- phase shift

$w$----- distance between the two fringes in the detecting field

When the positioning table feeds forward, the interference fringes will move up or down. When the direction of the sensor motion is opposite to that of the fringes, the minus sign is chosen in expression (3). On the contrary, the plus sign is chosen. If $a^{\prime}=0.83 \mu \mathrm{m}, \Delta w=10$ $\mu m$ and $w=10 \mathrm{~mm}$, then $d a=0.83 \mathrm{~nm}$.

\section{Fringe phase shift experiment}

\subsection{Precision positioning system}

A precision positioning system with grating interferometer displacement measuring device is developed. The whole system is composed of a precision positioning linear table, a set of precision grating interferometer device, a fringe phase-shift device and a computer control system. The block diagram of the system is shown as Fig. 3 and the photo of the positioning system is shown as Fig.4. The displacement of the precision positioning table is converted into pulses by the grating interferometer and the pulses is counted by counter and finally they go into industry computer.

The precision positioning linear table is the PLS-85 DC linear platform, a product of PI of Germany, with $50 \mathrm{~nm}$ increment. The core part of the precision grating interferometer device is a high precision diffraction grating with 300 lines per millimeter (or space $3.33 \mu \mathrm{m}$ ). The fringe phase-shift device, whose precision accuracy is $1 \mu \mathrm{m}$, is a linear driven by voice coil motor and controlled by Elmo controller.

\subsection{Experiments method}

oving slot Photo sensor

In order to validate the stripe phase shift method on displacement measurement, some positioning experiments with laser interferometer as measuring reference has been done on the precision positioning 


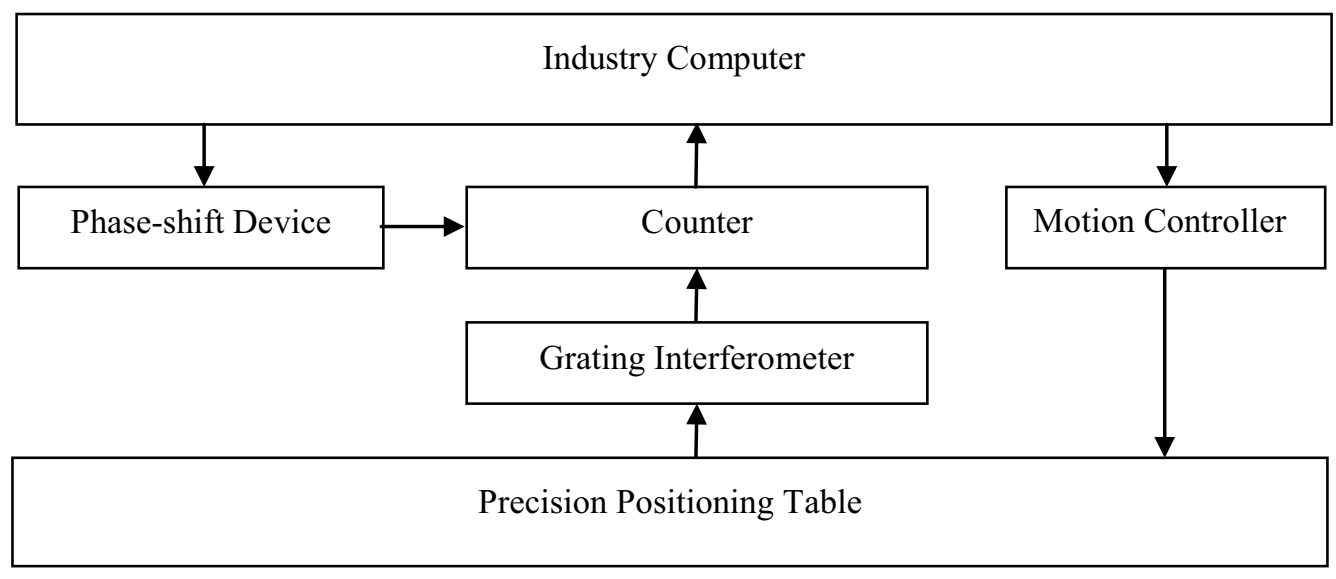

Fig. 3. Block diagram of positioning system

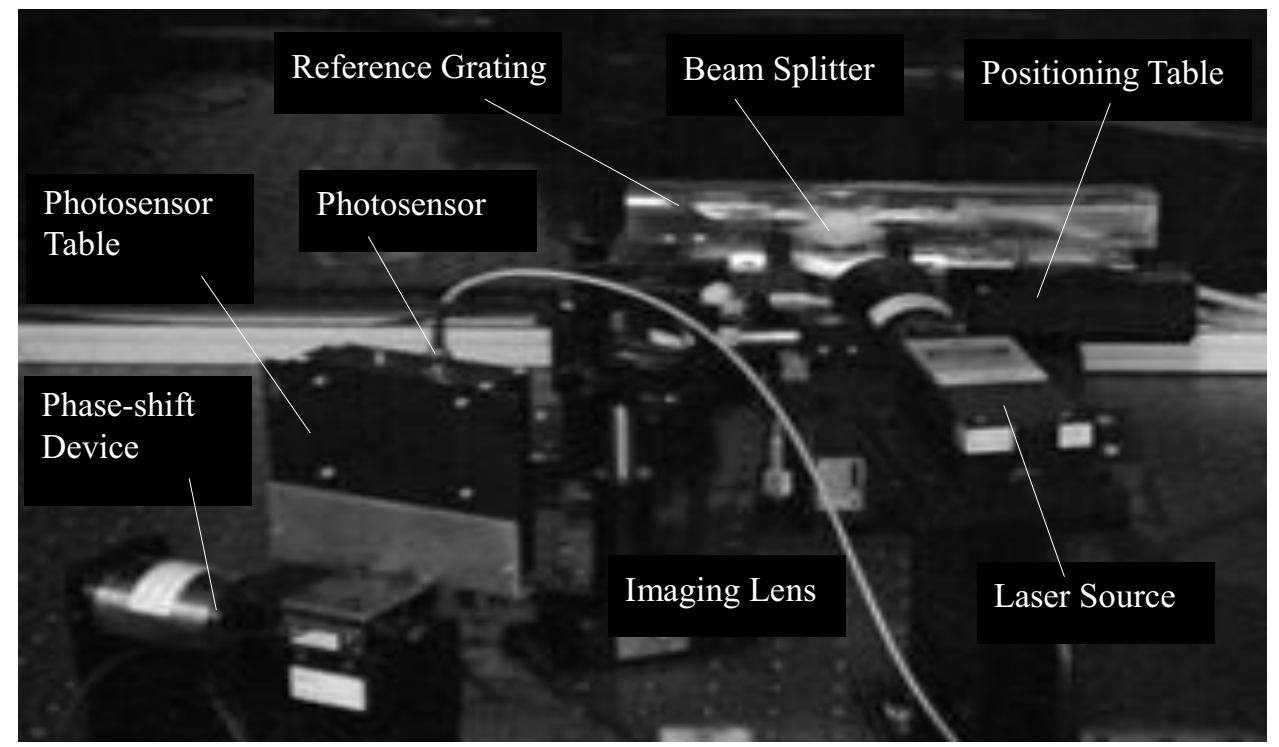

Fig. 4. The photo of the positioning system

system introduced above. The grating space of grating interferometer is $0.83 \mu \mathrm{m}$, with second order of interference fringe $(m=2)$ and $w=10 \mathrm{~mm}$.

The positioning point is selected at the point of $4 \mathrm{~mm}$ in the experiments. For example, in the $4 \mathrm{~mm}$ experiments, $4000 \mu \mathrm{m} / 0.83 \mu \mathrm{m}=4819.3$ pulse. Therefore, the positioning error of traditional method is $0.23 \mu \mathrm{m}$ in theory. In the experiments of new stripe phase shift method, after receiving 4818 pulses, the photo electric sensor will move and the positioning error of new method is $0.09 \mathrm{~nm}$ in theory. Actually, the error is bigger than $0.09 \mathrm{~nm}$ greatly because of the positioning errors of linear table itself and other factors.

Measurment Results of Experiments

\begin{tabular}{c|cccc}
\hline & \multicolumn{2}{|c}{ Traditional Method } & \multicolumn{2}{c}{ Phase-shift Method } \\
& Positioning & Error & $4000.142 \mu \mathrm{m}$ & $142 \mathrm{~nm}$ \\
& $3999.835 \mu \mathrm{m}$ & $165 \mathrm{~nm}$ & $4000.097 \mu \mathrm{m}$ & $97 \mathrm{~nm}$ \\
2 & $3999.791 \mu \mathrm{m}$ & $209 \mathrm{~nm}$ & $4000.122 \mu \mathrm{m}$ & $122 \mathrm{~nm}$ \\
3 & $3999.685 \mu \mathrm{m}$ & $315 \mathrm{~nm}$ & $4000.105 \mu \mathrm{m}$ & $105 \mathrm{~nm}$ \\
4 & $3999.843 \mu \mathrm{m}$ & $157 \mathrm{~nm}$ & $4000.131 \mu \mathrm{m}$ & $131 \mathrm{~nm}$ \\
5 & $3999.912 \mu \mathrm{m}$ & $88 \mathrm{~nm}$ & & $119 \mathrm{~nm}$ \\
Average (after & & $177 \mathrm{~nm}$ & \\
digital filtering) & & & & \\
\hline
\end{tabular}




\subsection{Experiments results and discussion}

The contrastive results on the positioning experiments with traditional method and new method are shown in the table. It indicates that the fringe phase shift method will improve the positioning accuracy about $33 \%$.

There are errors in the positioning process:

\subsubsection{Displacement accuracy of the phase-shift mechanism}

The actual displacement error of the phase-shift mechanism is less than $\pm 0.5 \mu \mathrm{m}$. Therefore, when the phase shift displacement is $10 \mu \mathrm{m}$, the resolution error will be less than 10 percentage of the academic value.

\subsubsection{Space error of the reference grating}

The transfer function of the grating interferometer can be calculated in the case of the space error being used as input value ${ }^{[2]}$. Supposing a concentrated error $\Delta a$ is in the $x_{0}$ position of the reference grating, it will be distributed in the area with width from $\left(x_{0}-d\right)$ to $\left(x_{0}+d\right)$ by the grating interferometer $(d$, the width of the light beam that irradiates on the reference grating). Namely, the concentrated error $\Delta a$ will be reduced to $\Delta a / 2 d$. For the concentrated error is reduced many times, it has little influence on the positioning process.

\subsubsection{Other factors}

The other factors, including the distance accuracy between the two interference fringes and some environment factors such as vibration and temperature in the working field, will bring errors also. Especially, the vibration from the base of the system will often bring gross errors. The gross errors in the experiments (such as the traditional method 3 and 5 on the table of measurment results) should be eliminated by digital filtering.

\section{Conclusion}

A new ultra-precision displacement measuring method, based on fringe phase shifting method, for grating interferometer displacement measuring system is developed. The core parts of the system is a grating interferometer and a phase-shift device. When the signal phase is changed by moving the photoelectric sensor between the two interference fringes in the view field, the displacement equivalent converted from the photoelectric sensor will changed correspondingly. Therefore, the new method could be applied in positioning system: supposing the photoelectric sensor is moved $10 \mu \mathrm{m}$, (the traditional resolution is $0.83 \mu \mathrm{m}$ and the distance between the two fringes in the detecting field is $10 \mathrm{~mm}$ ), then the resulution of the positioning system will be $0.83 \mathrm{~nm}$, namely, the resolution is 1000 times than that of the traditional method in theory. The experiments using the new method have been done and the results show that it will improve the positioning accuracy about $33 \%$. Also, the errors in the positioning process with the new method are discussed in briefly. It indicates that the displacement accuracy of the phase-shift mechanism, the space error of the reference grating and other factors such as vibration will influence the displacement revolution of the positioning process.

\section{References}

[1]. Marek Dobosz. Analysis of tolerances in a grating interferometer for high-resolution displacement measurement [C]. Proceedings of SPIE, Vol. 3744, 1999, 253 261

[2] HAO D F. Error Transfer Function for Grating Interferometer[C]. Proceedings of SPIE, Vol. 1545, 1991, 261-265

[3] Valery Kiryanov. Laser nanointerferometry of displacement methods and means of measurement accuracy improvement[C]. Proceedings of SPIE, Vol. 3736,1999, 410 415

[4] D. Croft and S. Devasia. High precision stages for micro/nano-lithography [C]. Proceedings of SPIE, 1997, 3225, 68 75

[5] Yoshioka H, Kuroyama S, Sawano H and Shinno H. Sub-nanometer positioning with a high resolution laser interferometer [C], Proceedings of the 10th International Conference of the European Society for Precision Engineering and Nanotechnology, Vol 1, 2010, 404-407

[6] Kawashima, Kenji, Arai and etal. Development of coarse/fine dual stage using pneumatically driven bellows actuator and cylinder with air bearings [J], Precision Engineering, Vol. 34, No.3, July 2010, 526-533

[7] Kwon S, Wan Kyun Chung, and Youm Y. On the coarse/fine dual-stage manipulators with robust perturbation compensator [C], Proceedings of IEEE, International Conference on Robotics and Automation, Vol. 1, 2001, 121-126

[8] Kwon SangJoo, Chung Wan Kyun and Youm Youngil. Robust and time-optimal control strategy for coarse/fine dual-stage manipulators [C], Proceedings of IEEE, International Conference on Robotics and Automation, Vol 4, 2000, 4051-4056

[9] Shirinzadeh Bijan and Teoh Pek L. Dual position sensitive diode-based orientation measurement in laser interferometry-based sensing and measurement technique [C], Proceedings of SPIE, Vol. 4564, 2001, 98-106

[10] Wang Wei. Program Controlled Amplifier and Its Typical Application [J]. OPTICS AND PRECISION ENGINEERING, Vol.6, No.2, 1998, 89-94

[11] SHI L, HAO D F, QI X D. High Precision Photoelectric Diffraction Grating Ruling Engine [J]. Chinese Journal of Scientific Instrument, 2001, 4(supplement), 103-104 